\section{IN BRIEF}

\section{$\Rightarrow$ ALZHEIMER DISEASE}

\section{Antibody targets apolipoprotein E to remove amyloid- $\beta$ deposits}

The antibody $\mathrm{HAE}-4$ specifically targets the apolipoprotein $\mathrm{E}$ (APOE) that is co-deposited with amyloid- $\beta(A \beta)$ in plaques and could remove $A \beta$ deposits without causing the adverse effects associated with $A \beta$-targeting antibodies, according to new research. Xiong et al. administered $\mathrm{HAE}-4$ to a genetically modified line of mice that express human $A P O E$ and naturally develop $A \beta$ deposits. Treatment with HAE- 4 began after the onset of $A \beta$ pathology, and mice that received HAE-4 had significantly less $A \beta$ pathology than mice that received a control antibody, suggesting that $\mathrm{HAE}-4$ can clear $\mathrm{A} \beta$. HAE-4 treatment was not associated with an increase in the number of microhaemorrhages, an adverse effect associated with $A \beta$-targeting antibodies.

ORIGINAL ARTICLE Xiong, M. et al. APOE immunotherapy reduces cerebral amyloid angiopathy and amyloid plaques while improving cerebrovascular function. Sci. Transl Med. 13, eabd7522 (2021)

\section{EPILEPSY}

\section{Blood purine levels as a biomarker in epilepsy}

Blood levels of the purine adenosine and its breakdown products could provide a biomarker of epilepsy and seizures, according to new research. In mice, blood purine concentration increased 2-3-fold after kainic acid-induced status epilepticus. Blood purine concentration was also higher in individuals with epilepsy than in healthy individuals. Measurements were taken with a chip-based system that uses blood from a finger prick and provides rapid results.

ORIGINAL ARTICLE Beamer, E. et al. Elevated blood purine levels as a biomarker of seizures and epilepsy. Epilepsia https://doi.org/10.1111/epi.16839 (2021)

\section{STROKE}

\section{Hourly risk score could alert clinicians to delayed cerebral ischaemia}

Delayed cerebral ischaemia (DCl) following aneurysmal subarachnoid haemorrhage is often detected too late for effective intervention. In a new study, Megjhani et al. applied machine learning to vital sign data from 310 patients with subarachnoid haemorrhage to generate hourly estimations of $\mathrm{DCl}$ risk. The resulting models were retrospectively applied to two different sets of test data and predicted $63.6-90.9 \%$ of $\mathrm{DCl}$ events $\geq 12 \mathrm{~h}$ before $\mathrm{DCl}$ had been diagnosed by clinicians. ORIGINAL ARTICLE Megjhani, M. et al. Dynamic detection of delayed cerebral ischemia. Stroke https://doi.org/10.1161/STROKEAHA.120.032546(2021)

\section{$\Rightarrow$ ALZHEIMER DISEASE}

\section{Distinct microglial profiles associated with amyloid} and tau pathology in Alzheimer disease

Microglia have different transcriptional profiles in Alzheimer disease (AD) depending on the type of pathology present, new research has shown. Gerrits et al. used single-nucleus RNA sequencing to study microglia in brain tissue from individuals with $A D$ and healthy controls. Two distinct $A D$-associated microglia profiles, AD1 and AD2, were identified. AD1 was associated with amyloid- $\beta$ pathology, whereas AD2 was associated with tau pathology. The findings could offer new targets for microglia-state-specific therapeutic interventions for AD.

ORIGINAL ARTICLE Gerrits, E. et al. Distinct amyloid- $\beta$ and tau-associated microglia profiles in Alzheimer's disease. Acta Neuropathol. https://doi.org/10.1007/ s00401-021-02263-w (2021)

\title{
Elevated risk of COVID-19 in people with dementia
}

Individuals with dementia have an increased risk of contracting and experiencing a poor outcome from COVID-19, according to new research published in Alzheimer's \& Dementia. The findings underline the need to develop strategies to protect patients with dementia from SARS-CoV-2 infection while avoiding the potentially detrimental effects of social isolation.

"Previous studies by others showed that the altered blood-brain barrier in people with Alzheimer disease $(\mathrm{AD})$ predisposes them to viral and bacterial infections," explains corresponding author Rong Xu. "In addition, the memory impairment associated with dementia might interfere with the patient's ability to adhere to preventive measures for COVID-19, such as social distancing, mask wearing and hand sanitizing."

$\mathrm{Xu}$ and colleagues analysed de-identified, population-level electronic health record data from $61,916,260$ individuals in the USA aged $\geq 18$ years, $1,064,960$ of whom were recorded as having dementia. The researchers used these data to examine the impact of dementia on the risk of developing COVID-19, and also on the probability of hospitalization and death as a result of the condition.

The analysis showed that people with dementia had a twofold increased risk of contracting COVID-19. The odds were highest in people with vascular dementia (adjusted OR (AOR) 3.17), followed by presenile dementia (AOR 2.62) and $\mathrm{AD}$ (AOR 1.86). In addition, dementia was associated with a greater likelihood of hospitalization and death as a consequence of COVID-19. The strong link between COVID-19 and vascular dementia indicates a possible role for pre-existing cerebrovascular pathology in SARS-CoV-2 infection.
The researchers also found that, among people with dementia, Black individuals had a higher risk of COVID-19 than white individuals, and were more likely to be hospitalized or die from the condition. These findings are consistent with COVID-19related racial disparities that have been observed in the wider population.

$\mathrm{Xu}$ and colleagues noted that people with dementia often have comorbidities that are risk factors for COVID-19, such as hypertension, cardiovascular disease, obesity or type 2 diabetes. Moreover, many patients with dementia reside in nursing homes, which tend to be hotspots for SARS-CoV-2 infection. However, the correlations between COVID-19 and dementia remained statistically significant after the researchers had controlled for these potential confounding factors.

"Prior evidence indicates a bidirectional relationship between viral infections and dementia, whereby people with dementia have an increased risk of viral infection, and a poor immune response to infection places individuals at an increased risk of dementia," comments Xu. "Further research is warranted to understand the underlying mechanisms - both biological and socioeconomic - of the increased risk of COVID-19 in patients with dementia, and to determine whether SARS-CoV-2 infection accelerates cognitive decline or triggers dementia."

Heather Wood

ORIGINAL ARTICLE Wang, Q. et al. COVID-19 and dementia: analyses of risk, disparity, and outcomes from electronic health records in the US. Alzheimers Dement. https://doi.org/10.1002/ alz.12296 (2021)

RELATED ARTICLES Numbers, K. \& Brodaty, H. The effects of the COVID-19 pandemic on people with dementia. Nat. Rev. Neurol. 17, 69-70 (2021) Nolen, L. \& Mejia, N. I. Inequities in neurology amplified by the COVID-19 pandemic. Nat. Rev. Neurol. 17, 67-68 (2021) 